Original Research Paper

\title{
Basma: An Interactive IoT-Based Plush Toy for Arabic- Speaking Children
}

\author{
Hend S. Al-Khalifa, Hadil R. Faisal, Shuhd M. Rushdi, \\ Ghaida M. AlNawwar, Ghadah N. Al-Gumaei and Alaa A. Alabduljabbar \\ Department of Information Technology, \\ College of Computer and Information Sciences, King Saud University, Riyadh, Saudi Arabia
}

Article history

Received: 16-09 2018

Revised: $27-10-2018$

Accepted: 02-11-2018

Corresponding Author:

Hend S. Al-Khalifa

Department of Information

Technology, College of

Computer and Information

Sciences, King Saud University,

Riyadh, Saudi Arabia

Email: hendk@ksu.edu.sa

hend.alkhalifa@gmail.com

\begin{abstract}
A toy is an item mainly intended for children to play with. However, due to its important role in child development, it has evolved to encompass sophisticated educational and learning characteristics. Toy industry has evolved to provide these needs as digital interactive smart toys. Interactive toys combine traditional toys with additional computing capabilities using software and hardware components. This paper presents the design and development of an interactive toy system named "Basma" which interacts with a child in Arabic language. The system consists of two parts: The first one is a plush toy with a hardware component that is implemented using a low cost Internet of Things (IoT) kit. This part leverages the interaction between the child and the toy, allowing the toy to respond and talk. The second part is an iPad application connected to the toy via Bluetooth. It allows parents to customize the toy and provides the child with stories that embed moral and cultural values. The interactive toy system was evaluated using test scenarios and user acceptance.
\end{abstract}

Keywords: Interactive Toy, Internet of Things, Learning Settings, Arabic Language, Edutainment

\section{Introduction}

Toy computing has recently emerged as a concept that focuses on advancing the functionality of traditional toys, using mobile technologies. Toys are no longer simple physical items, but comprise additional computing characteristics provided using different software and hardware components (Rafferty and Hung, 2015).

The integration of modern toys with mobile platforms was appealing due to the spread of personal mobile devices such as smartphones and tablets, together with the portability and flexibility that allows them to support applications developed for a wide range of uses. Toys with this feature, in addition to sensory and/or networking capabilities, can be referred to as Augmented, Interactive or Smart Toys. This is due to the fact that they are able to detect and interact with various changes in their surroundings (Rafferty et al., 2015).

Research shows that toys can be used to help children learn more about themselves and their surroundings; they can learn to solve problems and develop various skills, these skills can be listed as follows (UC Davis Cancer Center and UC Davis Children's Hospital):
- Develop cognitive concepts: Through the enhancement of their memory and attention span, which can be achieved by problem solving, for example puzzle games, colors, numbers, sizes and shapes

- Develop language skills: Using story-telling and what is called "make-believe", can greatly increase their literacy development

- Develop social skills: Learning to take turns in playing, or to "play by the rules" can have a significant impact in enhancing the child's understanding of society as they grow

Some of the available smart toys in the market include: Fisher, Dino, Trobo, Loujee, Hello Barbie and My Friend Cayla. Fisher comes with a pack of smart cards; the child can show these cards to the toy to choose the activity he/she wants. Some provided activities are games, adventures, storytelling, informing the child of the bedtime and engaging with him/her in drawing activities (Smart Toy). Dino is a dinosaur toy connected to a cloud, which gives Dino the ability to answer questions, play games, tell stories, crack jokes, as well as improve the child's vocabulary and mathematical knowledge through 
its educational games (Cognitoys). Similarly, TROBO is a talking plush robot with storytelling application that excites children about science, technology, engineering and math. The plush robot reads stories for the child and answers the questions they ask to explain complex subjects, giving the child an education head start in a fun and interactive way (Kickstarter). On the other hand, Loujee is considered the first toy in the market that is able to process Arabic language (Formal and informal) to create interactive dialogues. This toy consists of an iOS application installed on an iPhone or an iPad. The device is then inserted inside a stuffed toy, such that the device's screen will be visualized as the toy's face (Loujee.com). Two other toys specifically designed for girls are Hello Barbie and My Friend Cayla. Hello Barbie is an interactive doll that can process natural language as to have conversations and tell jokes and stories. It can be updated continuously as it is connected to $\mathrm{Wi}-\mathrm{Fi}$ (ChipChick). Cayla is a doll that connects via Bluetooth to a corresponding application. Cayla can talk, interact with the child and answer any question, from Math questions to everyday activities. Cayla can read stories played on the application and talk about the photo albums viewed there. The application also provides games (My Friend Cayla).

As we can see, toy industry is flourishing and many new technologies have been used and integrated to cover different aspects of childhood education and development. However, toy makers have only considered their culture and their values creating a gap for Arab children.

The goal of this project is to engage our Arab children in the new technological era and prepare them for the evolving world we live in. This can be achieved by teaching them our core values and Arabic language by employing interactive toys. Therefore, we can frame the contributions of our work as follows:

1. Utilization of the Internet of Things to build an educational system for Arabic speaking children

2. Introducing a child companion that interactively entertain and encourage cultural and moral behaviors

The rest of the paper is organized as follows: Section 2 sheds the light on previous research attempts of building interactive toys. Section 3 describes our system design and implementation. Section 4 presents our evaluation and test scenarios. Finally, the paper concludes with project limitation and future directions.

\section{Previous Work}

There has been some research on ways to create educational interactive toys for children as to improve their cognitive abilities and wellbeing. These contributions were designed to target a specific area, e.g. language learning, physical activity or cognitive development. This section will briefly discuss some previous research done to combine technology with stuffed toys as to create educational interactive experiences for children.

I-Eng (Fig. 1) is an interactive toy designed for children whose ages are 3-5 years. The aim of this toy is to help children learn a second language. The toy consists of three main parts: A plush toy that can talk, tangible objects and a storybook that works as a storage for the objects and contains the essential expressions. When the child shows an object to it, the toy recognizes the object and tells sentences related to it and based on the context, it asks the child about other related objects. As this interaction continues between the child and the toy, the child practices new vocabulary. Therefore, this provides an interesting learning experience based on the concept of "learn by doing". The talking toy prototype is comprised of Arduino and processing unit, the Arduino board with NFC-shield is embedded inside the plush toy. This board connects to a laptop with the processing code. The plush toy embeds also an LCD monitor and Bluetooth speakers, which receives signals from the laptop through the processing unit and then responds by making sounds and facial expressions on the monitor (Jeong et al., 2015).

Leal Penados et al. (2010) developed a toy that increases children's Physical Activity (PA), in the age 48 years, based on four encouraging factors: Fantasy, social interaction, overcoming physical and cultural barriers and other inspirational factors (such as curiosity, control and challenge). The developed cuddly toy, "Gum", senses the child's PA as it comes with a pocket to be attached to the child's waist. A Gum gets healthier as it gets a greater amount of PA daily, lighting more stars on its ears. The Gum, in addition, comes with food items (objects with an RFID tag), to be scattered around the house. When the child has been inactive for a long period, Gum will speak and ask for a specific item to eat, letting the child take the toy to where that item is placed (Fig. 2). The toy comes with a short story about the Gums, a parent's guide and a charger. Motion detection in this project was physically implemented using a twoaxis accelerometer (Leal Penados et al., 2010).

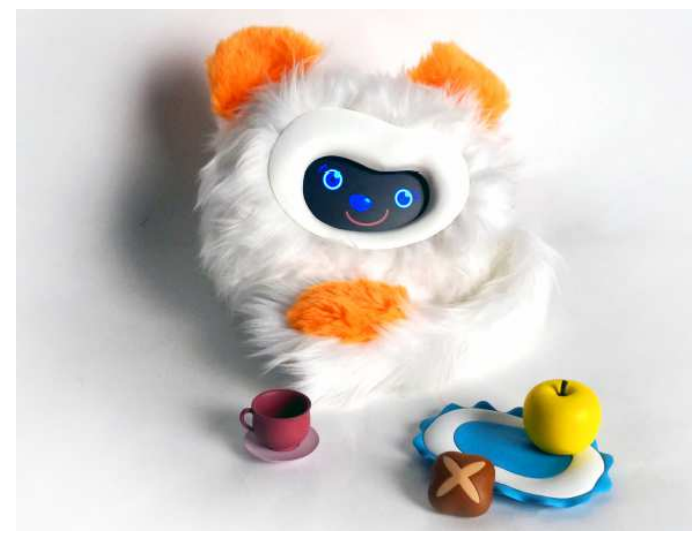

Fig. 1: I-Eng Toy (Jeong et al., 2015) 


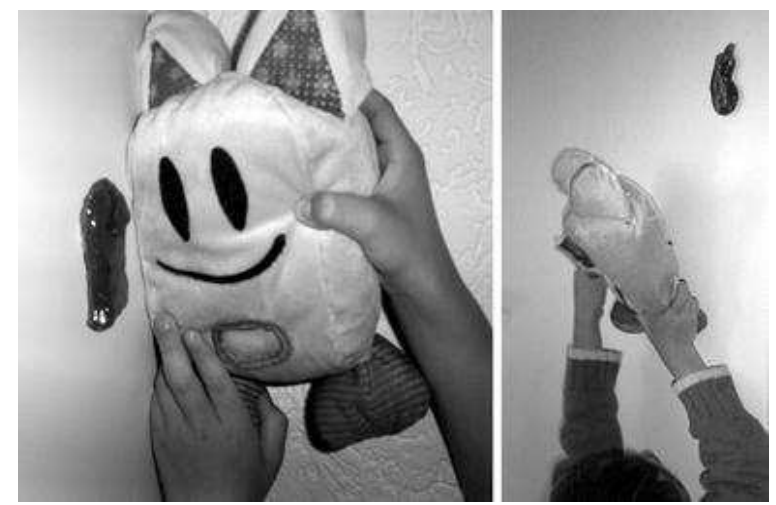

Fig. 2: Gum sensing a food item (Leal Penados et al., 2010)

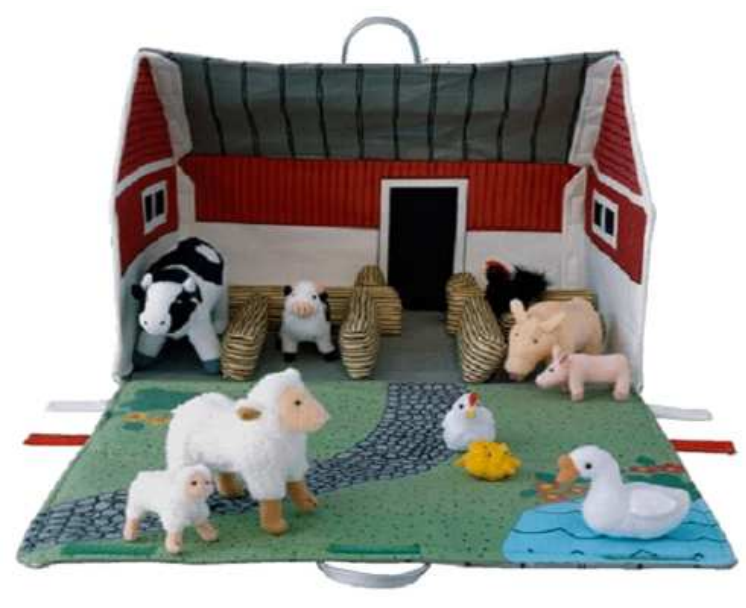

Fig. 3: User Interface of StoryToy (Fontijn and Mendels, 2005)

StoryToy (Fontijn and Mendels, 2005) is an interactive storytelling toy for children at the age between 2-6 years. It is an environment designed as a farmhouse (Fig. 3) with multiple stuffed animals characters that can tell stories. The system has three levels of operation: Free play, reactive play and story play. Which level is chosen can be specified by the location of the duck inside the farm. One thing that is special about this system is that multiple animals can work together to tell a story, react to each other and have conversations. In free play mode, the technology is turned off and the child can simply play with the stuffed toys. While in reactive mode, only audio is used to support the child interaction, such as playing the animal's sound when the child picks it up, or telling expressive sentences about their emotions. The main mode is story play, here the system tells a story in one of two types: Linear and branched story. In linear story, the narrator tells the story and at the end of each line, it mentions one of the animals and asks the child to pick it up and it progresses when the child picks the right animal. In branched stories, the child engages more and makes decisions in the story, by choosing to pick one animal or place it in a chosen location. When testing the system to explore the suitability of the different levels for children of different ages, reactive play was most popular with the youngest children. Linear stories were more enjoyable for children at age 3 , while branching story mode provided the most enjoyment for older children (4-6 years) since it offered them moments to think and reflect their opinions. The system appears magical to the child when he/she doesn't notice any technical parts visible in the interaction. This was implemented by embedding a motion sensor in each of the stuffed animals, these sensors are connected to a wireless transmitter. All sensor events are communicated to a receiver connected to a PC running a java program, the program translates these signals and sends the right audio response back to a wireless speaker inside the farm (Fontijn and Mendels, 2005).

On the other hand, SAGE (Storytelling Agent Generation Environment) is an authoring tool allowing children to design and interact with their own storytellers. It is a personal conversational storytelling system that resembles a Sage, a wise person, as it offers a story that is related to the speaker's concerns or experiences. Its goal is to enable children to learn about storytelling and technology and explore their inner world (Umaschi, 1997). Sage storyteller is embodied in an interactive stuffed animal to offer emotional engagement. It gives verbal and non-verbal feedback, such as the stuffed rabbit ears go up when it becomes a listener. The toy is connected to the SAGE Storyteller software on a Macintosh computer. Children input information through the keyboard (Umaschi, 1997).

Similarly, the Smart Teddy Bear is an audio-book player presented as a teddy bear with visual recognition capability. It assists children in their social-emotional growth. When a child wants to listen to a story, he/she opens any page in the story book in front of the Teddy Bear. Using an attached camera, the system automatically recognizes the book based on the pictures and plays the corresponding audio story. This toy is especially suitable for children from 3 to 5 years old (Pham et al., 2013).

Another teddy bear was upgraded with an embedded system to react on feelings. It consists of a teddy bear that has an embedded system that connects to a webcam and a speaker. The webcam live streams video that gets analyzed by a personal computer. It, then, tries to detect the child's facial expressions and consequently play a song to the child. The webcam and the live-streamed video also provides parental monitoring of the child (Goula-Dimitriou and Dasygenis, 2016).

Contrary to that, Dolltalk records the child's story and plays it back using a different voice pitch, representing the story character. The toy allows the child to tell his/her own stories, hear it in somebody else's voice and critic it. This improves the child's creativity in narrative elaboration. Dolltalk (Fig. 4) looks like a stage and two dolls. When the child removes the dolls from the stage, recording begins. Playback starts as the dolls are placed on the stage again (Vaucelle and Jehan, 2002). 
Cuddly is a mobile phone application that gets embedded in various soft objects to provide richer interactions with the objects. Using the phone's camera and flashlight (LED), it detects the surrounding brightness. When the user inserts the mobile phone into a soft object and presses the object, the brightness decreases. Cuddly, then, provides visual and auditory feedback based on this information. Furthermore, two or more mobile phones can be connected, creating a multiuser interaction (Low et al., 2013).

Some applications give voices based on the toy that the phone is inserted in, e.g., dog barking for a toy dog. In addition, the application displays visual faces on the screen. The animated character closes its eyes when the soft object is pressed. In addition, a gaming application (Fig. 5) has been designed to make a character jump higher as the brightness value decreases. Another application plays music when the phone is inserted into a pillow. The compression strength is measured as the user presses the pillow, different music is played and the phone's screen color changes accordingly. The phone inside the pillow can also act as an alarm clock. First, the user sets the time as any other alarm clock and then inserts it into the pillow. The alarm rings and vibrates at the time set. The alarm will stop when the user lifts his head up and will ring again as soon as the user lies back down (Low et al., 2013). The application can also leverage Bluetooth connectivity in the mobile phone to create a toy interaction with several devices. For example, the phone can be connected to a tablet and inserted into a pillow. As the user presses and punches the pillow, the character on the tablet takes a punch. Bluetooth can also be used for storytelling. Multiple users can play together by creating a shared conversation between toys. As two phones are inserted in two different soft toys and pressed, each will play a recorded script like telling a story (Low et al., 2013).

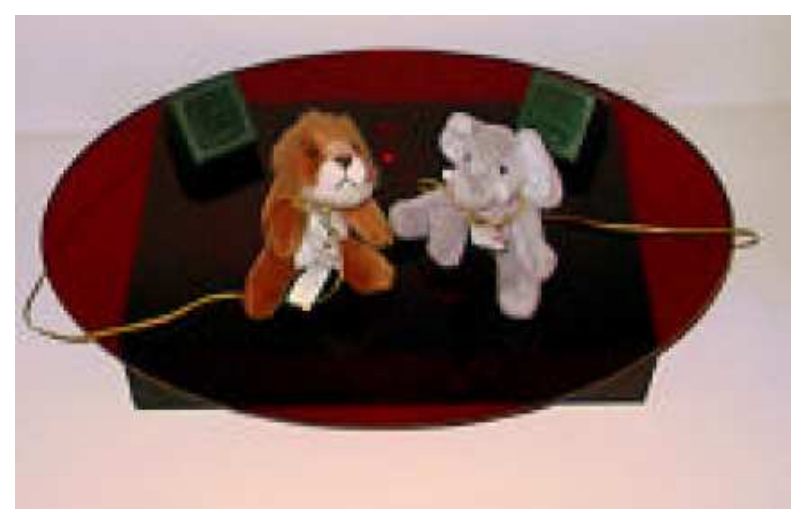

Fig. 4: Dolltalk (Vaucelle and Jehan, 2002)
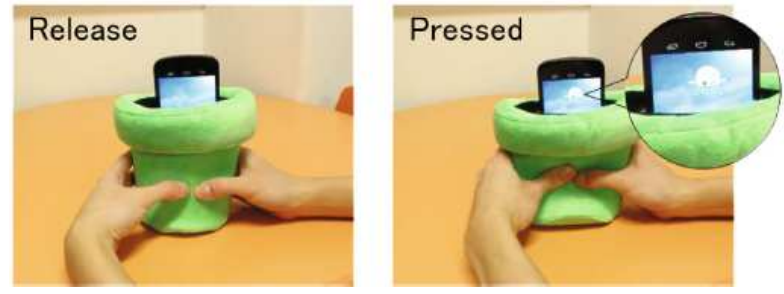

Fig. 5: The gaming application (Low et al., 2013)

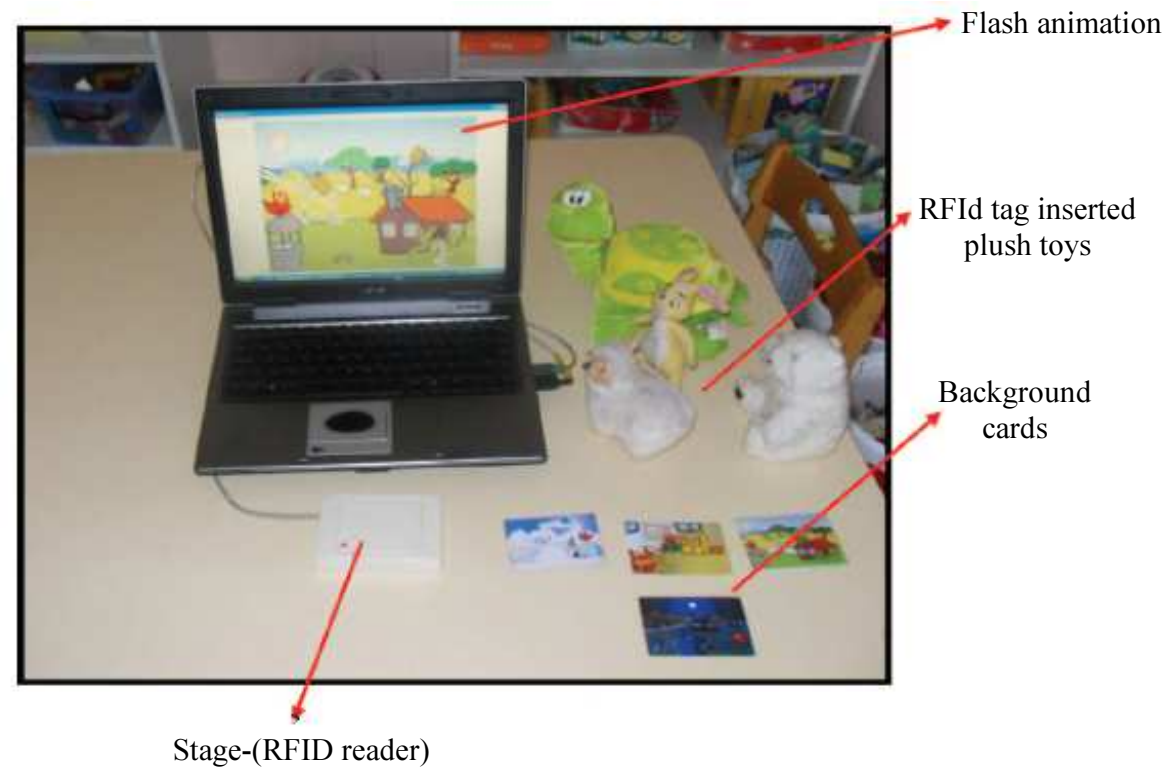

Fig. 6: StoryTech components (Kara et al., 2014a) 


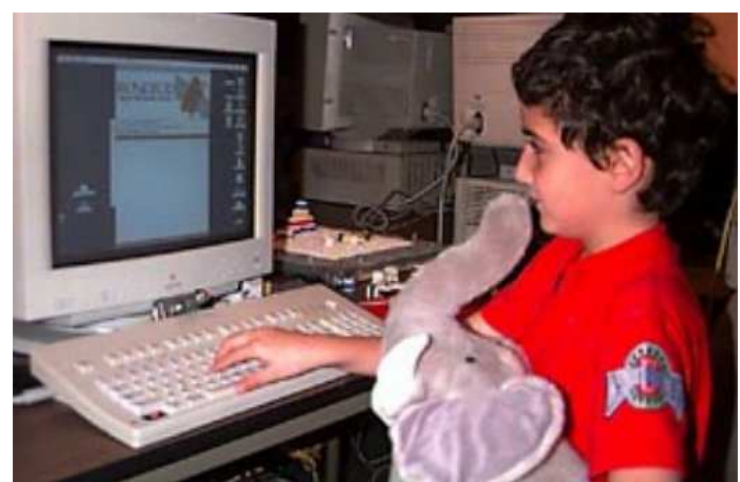

Fig. 7: Child writing a story using Rosebud (Glos and Cassell, 1997)

Likewise, StoryTech (Fig. 6) offers several computerbased background graphics, computer-generated animal characters with their corresponding stuffed toy, background cards and a receiver panel. StoryTech allows children to use various background cards and place animal characters on the stage to make their own story. When an animal is placed on the stage, the computer displays related backgrounds and characters and plays the actual sound of that animal. Children are expected to start telling their own story based on what the screen displays (Kara et al., 2014a).

Also, Rosebud (Fig. 7) enhances children storytelling skills while interacting with a computationallyaugmented toy. Each stuffed animal holds an infrared transmitter in order for the computer to identify the toy. The system links children's stories to their toys. Each toy has its own stories that accumulate over time. When the child writes a story, the system generates story-specific feedback and encouragement based on data extracted from the narrative. When a new toy is introduced to Rosebud, it asks for the toy's name and if the toy "knows" any of the previously used toys. If Rosebud knows anything about the toy, it will provide it to the child, e.g., the computer will tell about a platypus if introduced to one. In addition, Rosebud encourages children to enhance their stories. It creates a "storybook" of saved stories. Children can edit, revise, collaborate and share stories in the storybook (Glos and Cassell, 1997).

As we can see from all the above stated research, they were designed only to entertain, or target a specific area, e.g., language learning, physical activity or cognitive development. None has provided a complete system that would act as a child companion, entertaining and teaching the child, not only facts and science, but also culture values and morals. This gap will be bridged by our project "Basma".

\section{Basma Rationale}

The curriculum provided in kindergarten schools in the Arab region especially in Saudi Arabia, focuses on
Arabic Alphabet, numbers, morals and cultural behaviors. Every subject in kindergarten has different methods to be taught with and introduced to a child. Teaching methods are varied, the most prominent way is learning by playing. Playing helps children learn, gain confidence and develop socially and cognitively (Cagiltay et al., 2014). Storytelling is also a major teaching method as it entertains, develops children imagination and oral language and builds up the child attitude in various social contexts (Pham et al., 2013; Kara et al., 2014b). Another way of teaching is by using songs, where they also have significant efficacy on a child's learning. In addition, children memorize supplications and other needed subjects by repetition.

Children's education is not limited to kindergarten. Parents are the main aspect of teaching and guiding their children. There must be collaboration between kindergarten and a child's parent to make sure that the education process is complete.

Regardless of the educational environment and proposed materials, motivation and encouragement are the most important cornerstones to improve the child's learning skills. Various methods and theories are developed to motivate children and have shown great results. One of the most effective ways to motivate children is what is called a "reward chart", where the child could reach the final goal or collect a specific number of some object-stars for example-. In addition to the tangible or physical means of encouragement, verbal encouragement is as important. Parents and teachers must encourage and motivate children by using words and phrases.

Children do not learn any behavior by giving them orders; theory is not enough for children to change their behavior. Other methods are preferred to teach a child a particular behavior. The most important one is reminding them in a variety of ways.

Given these characteristics of kindergarten education, Basma will provide simple and clear content similar to what is being taught for the specified age group and in their mother tongue.

\section{System Design}

Basma system consists of components: Hardware (the plush toy) and software (the iPad Application). This section will discuss Basma user characteristics, functional requirements, hardware specification, user interface and story design.

\section{User Characteristics}

Basma system has two users: The parent and the child. The parent is responsible for customizing the toy and creating an avatar of the child. In addition, the parent can add actions and reminders and track the child progress and rewards. Since these tasks are done by 
interacting with the Application installed on the iPad, the parent's technical expertise should be medium to high. However, no advanced computer skills are required except for the knowledge of how to download applications and navigate through the software. As for the language, parents should know how to write and read Arabic language, since the application uses Arabic. Nevertheless, no restrictions are imposed on parents' education level and age.

As for the child, the targeted age group is 3-6 years old, preschoolers and kindergarteners. The expected technical experience of children is low. The child should be able to understand simple Arabic language words and phrases. The child interacts with Basma toy in addition to the Application. Application interaction is to allow the child to invoke specific functions in Basma's system. However, their interaction with Basma toy will be through touch sensors embedded in the toy's hands.

\section{Functional Requirements}

Based on Basma's user characteristics, we describe here the functional requirement for each user.

For children:

1. Basma system should be able to tell a story selected by the child

1.1. The iPad Application shall display a list of the available stories

1.2. The iPad Application shall allow the child to select a story

2. Basma toy should be able to identify supplication objects

2.2. Basma toy shall be able to detect and identify a pre-tagged object using NFC reader

2.3. Basma toy shall say the supplication related to an object

3. Basma toy should remind the child of specific actions (e.g., brushing her teeth) at particular times set by the parent

3.2. 3Basma toy shall remind the child of the action

3.3. Basma toy will provide a response depending on the child answer

3.4. The system should reward the child based on her response

3.5. The system shall unlock a new story when the child completes a specific number of stars

For parents:

1. The parent shall be able to set up Basma system

1.1. The parent shall be able to set up a connection with Basma toy via Bluetooth
1.2. The iPad application shall display a parent interface

2. The parent shall be able to create a profile for his/her child

2.1. The iPad application shall allow the parent to create a profile for his/her child by entering her name

2.2. The iPad application shall allow the parent to build an avatar for his/her child by selecting from a predefined set of avatars

3. The parent shall be able to enable/disable reminders for a predefined list of actions (Morning/evening supplications, brushing teeth, kissing parents and bedtime)

3.1. The iPad application shall allow the parent to view a predefined list of actions to be a reminder for her child

3.2. The iPad application shall allow the parent to enable/disable a specific action

3.3. The iPad application shall allow the parent to specify a time for each enabled action to alert his/her child in certain time

4. The parent shall be able to view the child progress

4.1. The iPad application shall display a chart of the children earned stars during the current month

4.2. The iPad application shall update the chart each time the child earns a new star

4.3. The iPad application shall reset the chart each new month and start over

\section{Basma Hardware Specification}

Basma toy (Fig. 8) is a stuffed interactive toy with an embedded Arduino controller and various connected shields and peripherals. Basma has an integrated LCD screen on her chest to display the child information, e.g., earned stars to show constructive feedback. These are displayed to increase the child sense of belonging to Basma. Collected stars are also displayed on the screen as to encourage the child good behavior. As the child continues to interact with Basma system, more stars will be gained and, when reaching a pre-defined limit, new stories in the application will be unlocked as part of the rewarding system. These stories will help the child to learn good morals and behaviors.

Basma takes input from the child to figure out if he/she has done a specific task. These inputs are passed by hand sensors as the child presses on them to answer yes, by pressing on the right-hand sensor, or no, by pressing on the left-hand sensor. In addition, Basma is connected to an NFC reader to allow it to recognize a set of pre-tagged objects and tell the child the related 
supplications. Furthermore, Basma uses speakers and hand sensors to effectively apply the techniques of "story telling" and engage the child in a story that teaches $\mathrm{him} /$ her virtuous behavior.

As for the external design, Basma looks like a little girl with fluffy long dress and smiley face. The color used is soft tons of white and pink, which pleases the targeted group, female children. Basma's dimensions are $73 \times 19 \times 10 \mathrm{~cm}$.

\section{User Interface and Story Design}

Figure 9 shows Basma's software interface hierarchy diagram (iPad Application) and how a user may navigate through the different interfaces. Basma software provides two panels: Parent panel, as shown in Fig. 10 and child panel, as shown in Fig. 11.

The parent panel provides the necessary functions to create and edit the child profile and set reminders. While the child panel consists of two main functions, which are: stories and supplications (a.k.a Athkar).

The story to be told by Basma was designed with the intention of delivering a message for the child. It has one main character who wants to compete against her friend by running a race through a forest, each goes on a separate way. At the beginning of the race, a small bird tells them that good manners are the most important aspect of winning - which can be considered as the main message the story is delivering-. Then a series of events happen, some of them require the child's decision to help the main character throughout the race. The story incorporates other characters that the main character meets as she goes on her way, mostly animal characters; as these can capture children's attention at this age. The story was designed by an expert in children education.

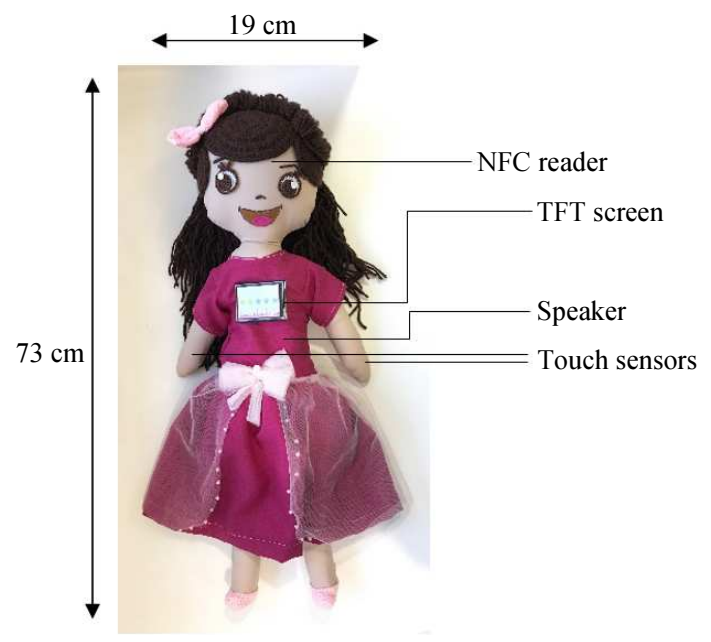

Fig. 8: Basma toy depiction

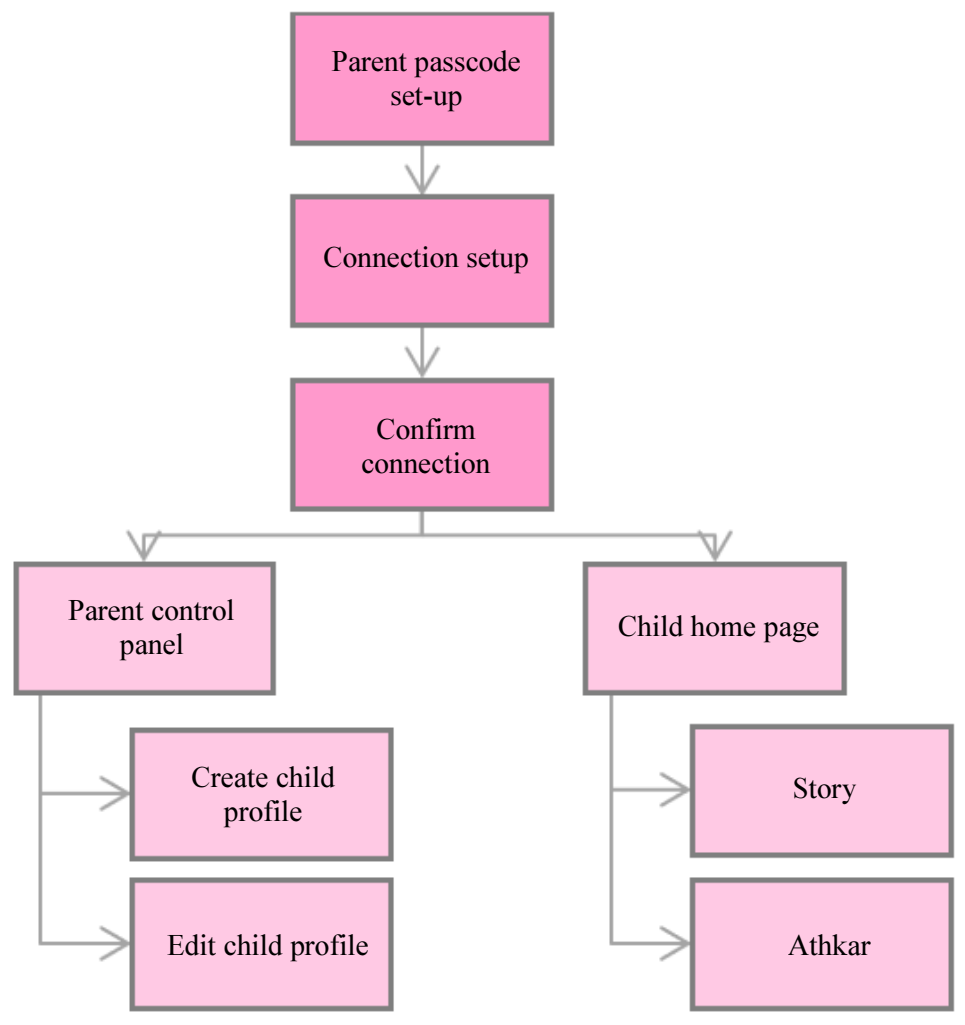

Fig. 9: User interface hierarchy diagram 


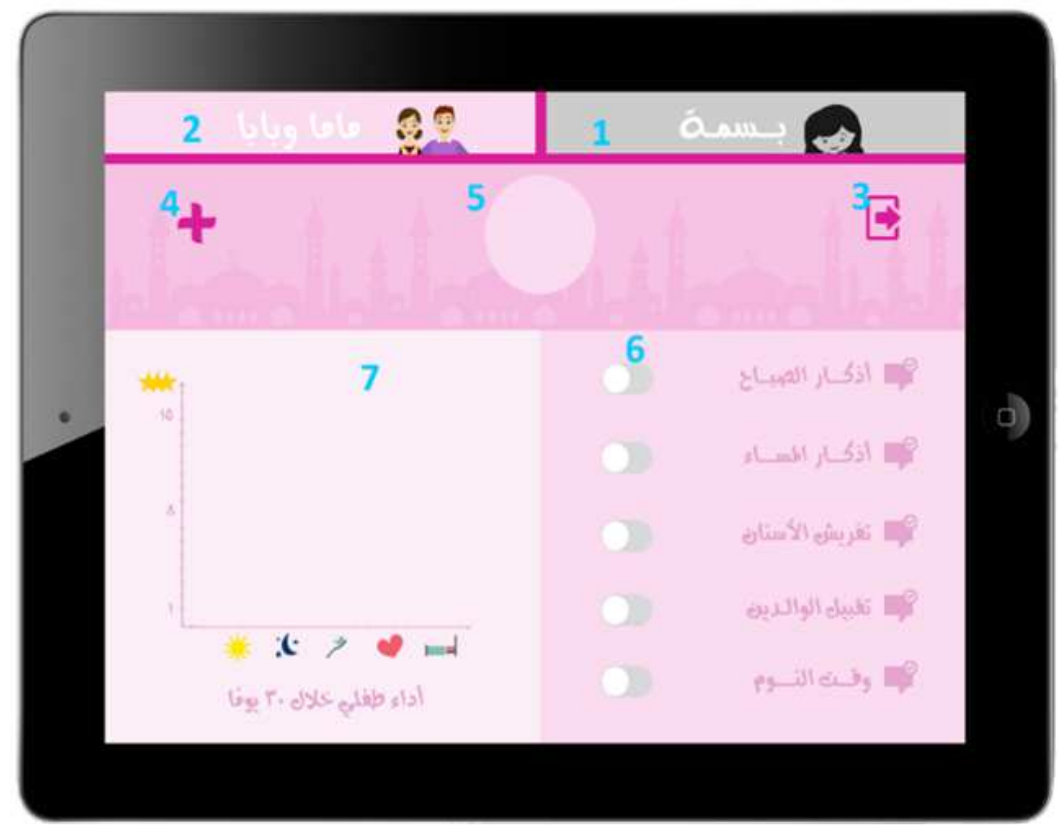

Fig 10: Parents' control panel page with the following buttons: (1) display child home page, (2) display parent control panel, (3) logout (4) create child profile page, (5) Image holder to display the child's avatar, (6) enable/disable the actions reminder and (7) A graph to view the child progress

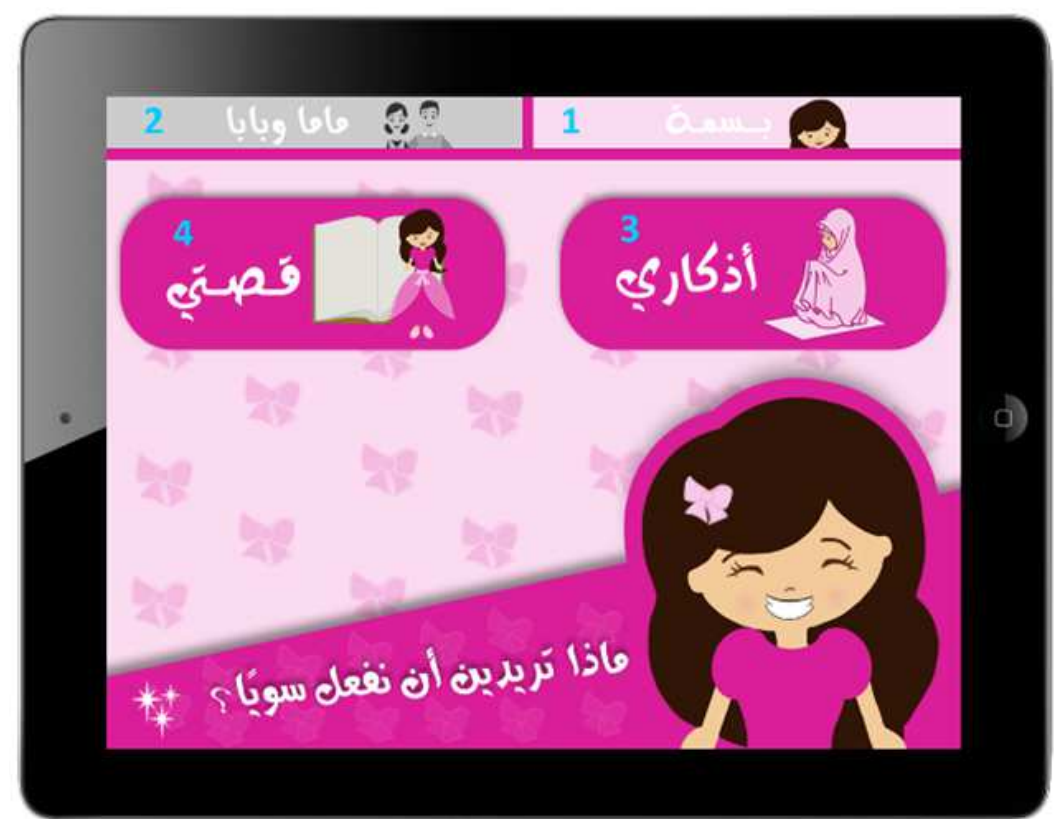

Fig. 11: Child Homepage with the following buttons: (1) display child homepage, (2) display parent control panel, (3) open the supplications page, (4) open the stories page

\section{System Implementation}

To achieve Basma's functionality, many electronic shields and components were used. Table 1 illustrates the used hardware components that Basma embeds. Arduino Uno is the controlling unit that connects and integrates all these components. Figure 12 shows a high-level block diagram explanation of how all components were connected together.

Basma system consists of programmed hardware components that are used as I/O channels, connected via Bluetooth Low Energy (BLE) to software 
components that provide content and manage interactions (Fig. 13). Figure 14 shows the possible action sequences that are supported by the hardware and software components. First, the parent sets up the system and sets the reminders. At the time of the reminder, the software sends a script to the hardware then the child interacts with Basma (Hardware). The results are transmitted wirelessly back to Basma Software for tracking. The child can activate the hardware components via the Software functions "Listen to a story" and "Listen to Supplications" to act accordingly.

Table 1: Hardware components specification

\begin{tabular}{|c|c|c|}
\hline Name & Description & Used for \\
\hline Arduino UNO & $\begin{array}{l}\text { A microcontroller board based on the } \\
\text { ATmega328P. }\end{array}$ & $\begin{array}{l}\text { It's the main component of Basma that } \\
\text { controls all other connected shield. }\end{array}$ \\
\hline $6 \times$ AA battery holder & $\begin{array}{l}\text { AA's for a 9V 3000-4000mAh power supply, or } \\
\text { rechargeable NiMH for } 2000 \mathrm{mAh} 7.5 \mathrm{~V} \text { supply. }\end{array}$ & $\begin{array}{l}\text { Used for charging Basma without being constantly } \\
\text { connected to a power source via a cable. }\end{array}$ \\
\hline 2.8" TFT Touch Shield & $\begin{array}{l}\text { TFT display is }(2.8 " \text { diagonal }) \text { bright and colorful } \\
240 \times 320 \text { pixels with individual pixel control. }\end{array}$ & Used for displaying the child's earned stars. \\
\hline Adafruit Feather M0 & Bluetooth Low Energy is the only wireless & Used for connecting Basma with the application \\
\hline Bluefruit LE & $\begin{array}{l}\text { protocol that is used with iOS without needing } \\
\text { special certification and it's supported by all } \\
\text { modern smart phones. }\end{array}$ & on the iPad. \\
\hline Adafruit PN532 NFC/RFID & Tool for any $13.56 \mathrm{MHz}$ RFID or NFC & Used for identifying the pre-tagged objects that \\
\hline Controller Shield & $\begin{array}{l}\text { application that reads and writes to tags and } \\
\text { cards. }\end{array}$ & hold the Athkar. \\
\hline Wave Shield & It adds a quality audio to an electronic project. & $\begin{array}{l}\text { Used as a speaker for Basma to tell stories, Thikr } \\
\text { and communicate with the child. }\end{array}$ \\
\hline $\begin{array}{l}12 \times \text { Capacitive Touch Shield } \\
\text { for Arduino - MPR } 121\end{array}$ & $\begin{array}{l}\text { The shield has } 12 \text { capacitive touch sensor holes } \\
\text { that can be gripped onto with alligator clip } \\
\text { cables. Each can detect when it has been touched. }\end{array}$ & $\begin{array}{l}\text { Used to get input from the child and facilitate } \\
\text { interaction. }\end{array}$ \\
\hline Alligator Clips & $\begin{array}{l}15 " \text { cables that have an alligator clip on both } \\
\text { ends. }\end{array}$ & Used to extend the range of the touch shield. \\
\hline
\end{tabular}

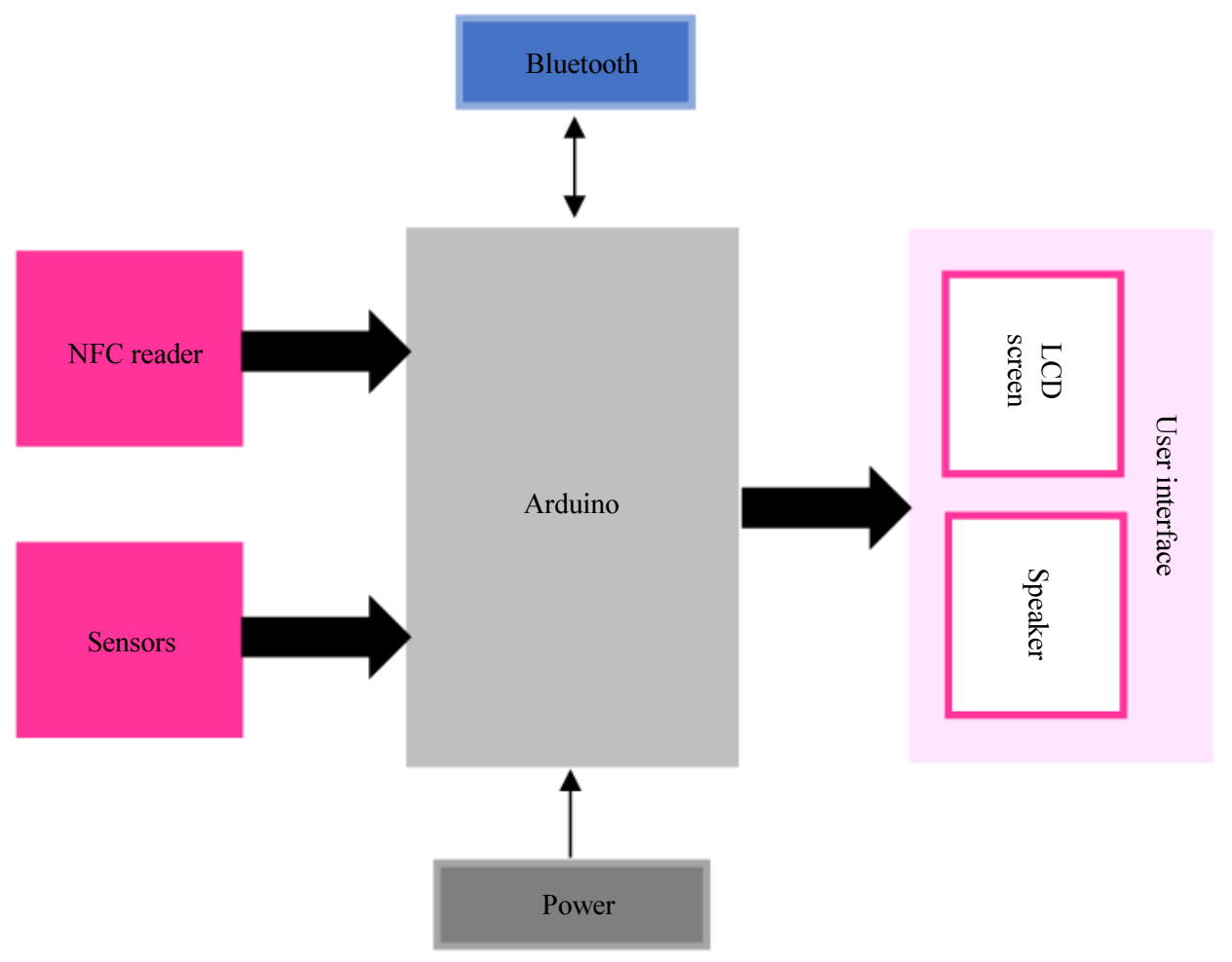

Fig. 12: Block-diagram for hardware component integration 


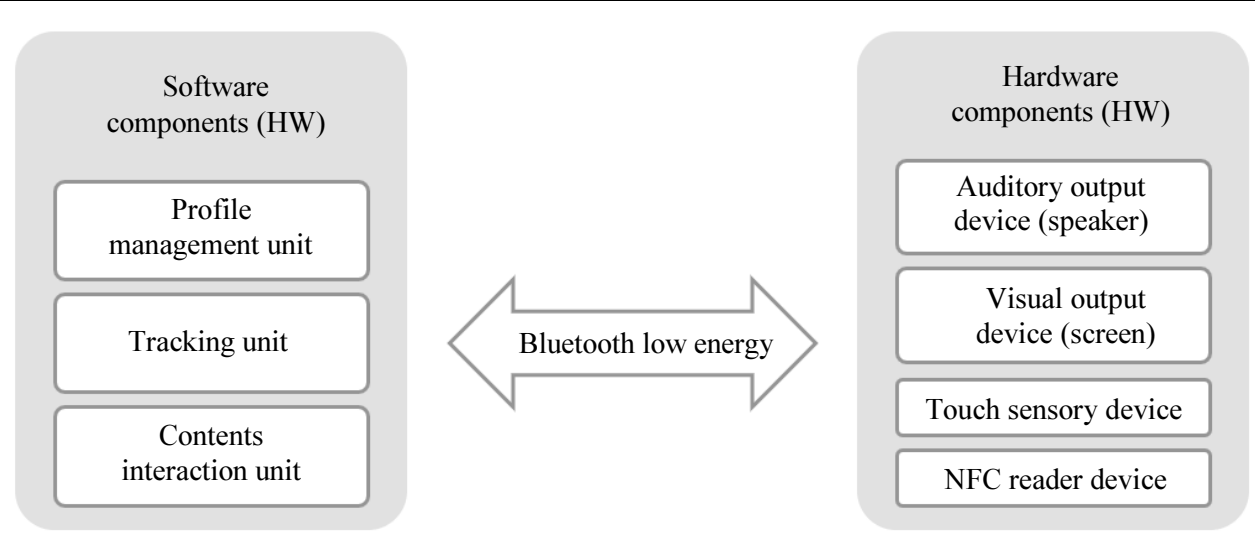

Fig. 13: Basma system configuration

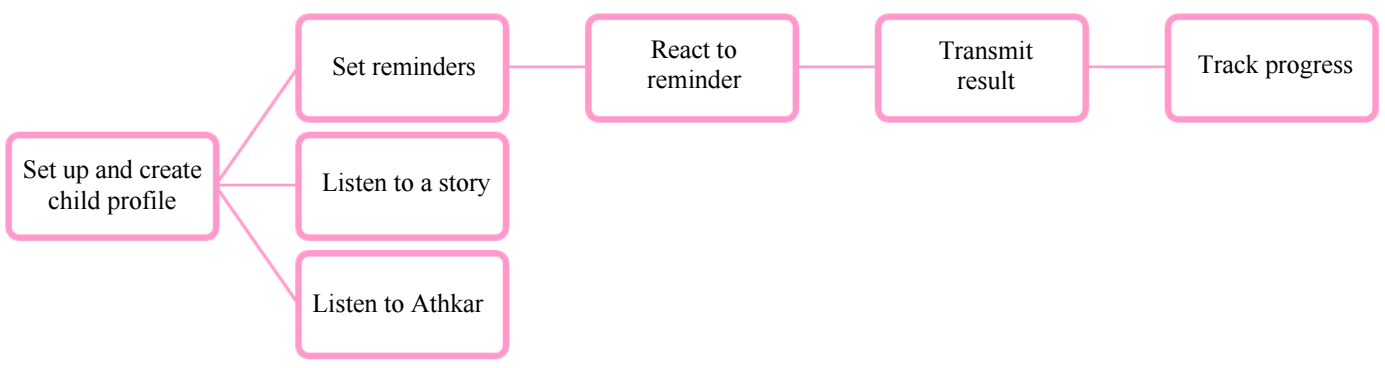

Fig. 14: Possible action sequences in Basma system

Table 2: Script commands

\begin{tabular}{lllll}
\hline Classification & Command & From & To & Parameters \\
\hline Wave shield activation & W: & iOS app & Arduino & File name \\
TFT shield activation & $\mathrm{S}$ & iOS app & Arduino & Number of stars to be displayed \\
Touch shield activation & T & iOS app & Arduino & - \\
Touch shield response & 0 or 1 & Arduino & iOS app & Touched Capacitor No. \\
NFC shield activation & N & iOS app & Arduino & - \\
NFC Shield Response & 0 or 1 & Arduino & iOS app & Object Detected No. \\
\hline
\end{tabular}

We have designed a complex system that composes two Arduino UNO, 4 shields and a Bluetooth Low Energy (BLE) chip. The main controllers are the two Arduino UNO. The first UNO provides output through the TFT Touch shield (screen), while the second UNO is responsible for controlling the Wave shield (speakers), the Touch shield (sensors) and the NFC Controller shield. Wireless communications are facilitated by Feather M0 Bluefruit LE through UART, which is connected to both Arduinos.

The software code running on the processing unit (UNO) is responsible for managing BLE connection, parsing scripts received from the mobile application and then activating and managing appropriate shields. Table 2 shows the script commands transmitted between the hardware and the mobile application for controlling the shields. After parsing the received script, the appropriate shield is activated and managed according to the received parameters.

As for the story, the previous sketches were used to design the scenes with Adobe Photoshop and Illustrator, as well as the characters throughout the story. As for the sounds, they were recorded using an iPhone, the voice was later edited using Audacity to change the pitch and make it suitable for Basma, give it more quality and be later playable on the wave shield according to its requirements. The story has interactive questions that appear on the iPad and the child can choose among three alternatives the correct answer. Also, the story incorporates the ability to change its events based on the child's decision. For example, a child can pick the east or west directions by using the hand sensors embedded in Basma toy, this is shown in Fig. 15.

\section{Evaluation}

\section{Test Scenarios}

User acceptance testing involves the contribution of different stakeholders who will be using the system; they will be giving some feedback and preferred adjustments. Basma project required the participation of two 
stakeholders; parents and children. Four parents have answered a questionnaire (provided in appendix A.1) to evaluate their observations on their children's behavioral changes and their connection to Basma. As for the children, they were also surveyed (appendix A.2), but with more friendly and appealing alternatives to choose from (smiley faces).

This section will present the different scenarios that were followed during the user acceptance testing for stakeholders, four parents and four children were tested during the following sessions.

The parent scenarios included the parent setting up a passcode and resetting it, adding a child and setting up a reminder and lastly using Basma for the first time with their child. In the first scenario, the parent was asked to add his/her child by typing their name and choosing an avatar. Afterwards, they were asked to set up a reminder for Evening supplications at 6:00 pm. This scenario revealed that the parents needed more instructions that were later added to the interfaces. The other scenario was intended to make children familiar with Basma's functionalities with the presence of their parents. Parents were asked to explain, in details, how the child should react to reminders and how she will be rewarded. They were also asked to explain the two buttons on the child homepage, stories and supplications and how each works. This session's evaluation was based on how much children have grasped the idea of Basma and the iPad application and whether parents found it easy to teach their children how to use Basma. Children were further evaluated alone on the next test scenarios. It was very clear that the child understood exactly how it worked and it was easy to use after their parents' instructions, which revealed a good indicator.

As for the child scenarios, it included listening and interacting with stories and using supplication objects. In the former, the child was asked to choose the first unlocked story and we observed her interaction, how much she was attracted to the characters and the colors and how excited she was to move to further scenes. When asked, the child said she was very impressed with the color and characters and very excited to finish the story. In the latter scenario, the child was asked to grab the supplication object, move it close to Basma's head and tell the supplication with Basma (Fig. 16). This session was evaluated based on the child's understanding of how much proximity is needed for Basma to narrate the supplications and on whether they learned the related supplication after narrating it multiple times.

\section{Discussion}

After surveying both stakeholders, taking feedback and children observations, it was clear that Basma was able to achieve its initial objectives successfully. Parents were able to deal with their own view quite smoothly and children were able to capture the idea of Basma easily and they were enthusiastic to do more actions and gain stars. Children who are between (3-4) years old have shown more interest in the object identification, listening to and repeating the supplications. On the other hand, older ages -(5-6) years old- were more interested in the stories and interacting with them.

Figure 17 shows some results of parents' questionnaire. These results show that the plush toy was the first thing that attracted children in Basma system and that emphasizing on upgrading its functionalities is what is expected in the next stage. Also, parents have shown a great interest in purchasing Basma for their children if it was launched in the market. Finally, children were enthusiastic about Basma and have shown a great interest and interaction with it. Figure 18 shows their answers on the questionnaire.

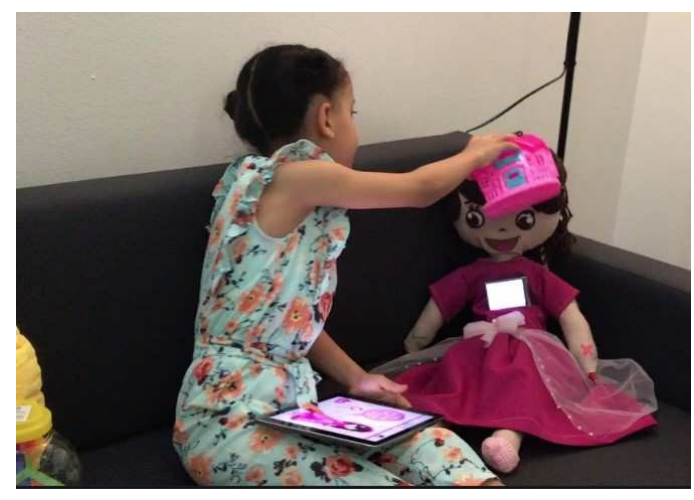

Fig. 15: Children using objects with Basma

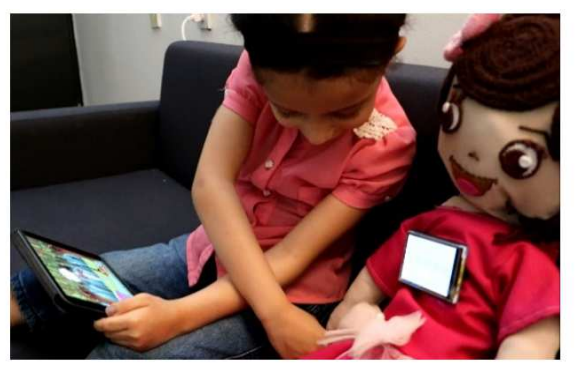

Fig. 16: Screenshot of the story (left) A child making decisions on the story events using the hand sensors (right) 
Would rewarding process help encourage your child to do more actions on time?

4 responses

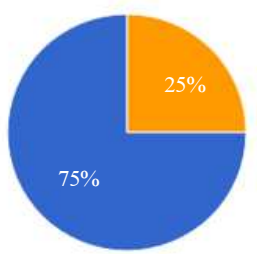

- Yes

No

If you wish the developers could emphasize more on one aspect of Basma, what would it be?

4 responses

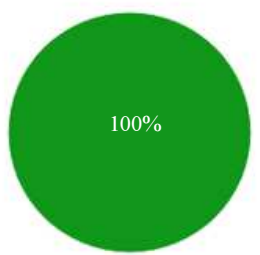

- Stories diversity

Athkar diversity

Reminders diversity

Plush toy capabilities and

functionalities

If Basma was launched in the market, would you buy it for your child? 4 responses

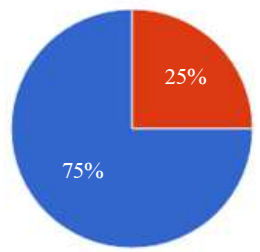

- Definitely

- Maybe

I'll give it more time be

develop and provide more

Which of those do you find your child more interested in?

4 responses

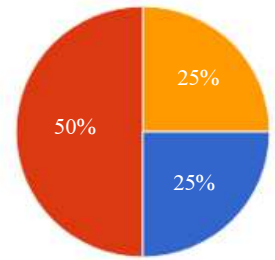

Interactive stories

- Object identification and Akhtar

Reminders and gaining more stars

Fig. 17: Results obtained from parent's questionnaire

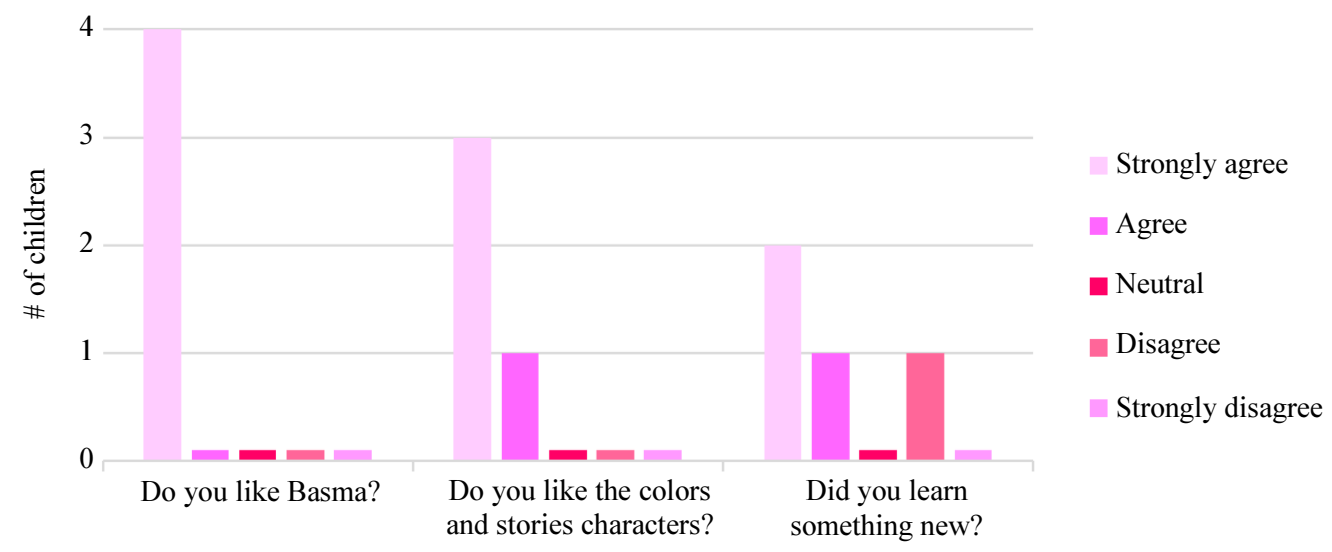

Fig. 18: Results obtained from children's questionnaire 


\section{Conclusion}

Although toy computing is relatively new to the technological revolution, a variety of interactive toys has dominated the market and showed good success and acceptance. However, none of those have provided the functions that this project focuses on in delivering a complete system that acts as a child companion that entertains and teaches facts and, more importantly, morals, in Arabic language. Basma has a potential of standing out in the field of interactive toys; due to the lack of such innovative edutainment solutions nationally. On the other hand, Basma can also stand out in its field internationally; as it delivers the novel mission of reviving the moral values that the local culture encourages.

\section{Acknowledgement}

We gratefully acknowledge Teacher. Khulood AlGumaei for her great support in conducting the interviews, writing us the stories and providing continuous feedback regarding children choices and preferences. We also acknowledge iWAN Research Group and The Engineering Innovation Center at King Saud University for supporting this project financially. Will also thank the efforts of the Deanship of Scientific Research at King Saud University as part of the Undergraduate Students Research Support Program (USRSP).

\section{Funding Information}

Deanship of Scientific Research at King Saud University as part of the Undergraduate Students Research Support Program (USRSP).

\section{Author's Contributions}

Hend Al-Khalifa: Makes substantial contributions in the development, writing and formatting of the manuscript, proofreading and giving final approval of the manuscript to be submitted.

Hadil Faisal, Shuhd Rushdi, Ghaida AINawwar, Ghadah Al-Gumaei and Alaa Alabduljabbar: Contribute in writing and formatting of the manuscript and the analysis, development and testing of the application.

\section{References}

Cagiltay, K., N. Kara and C.C. Aydin, 2014. Smart Toy Based Learning. In: Handbook of Research on Educational Communications and Technology, Spector, J.M., M.D. Merrill, J. Elen and M.J. Bishop (Eds.), Springer, New York,

ISBN-10: 1461431859, pp: 703-711.
ChipChick, 2015. Hello Barbie is the first interactive Barbie doll, with Siri-style tricks.

Cognitoys. How It Works. https://cognitoys.com/pages/about

Fontijn, W. and P. Mendels, 2005. StoryToy the interactive storytelling toy. Proceedings of the 2nd International Workshop on Gaming Applications in Pervasive Computing Environments at Pervasive, (CEP' 05).

Glos, J.W. and J. Cassell, 1997. Rosebud: Technological toys for storytelling. Proceedings of the Extended Abstracts on Human Factors in Computing Systems, Mar. 22-27, ACM, Atlanta, Georgia, pp: 359-360.

DOI: $10.1145 / 1120212.1120433$

Goula-Dimitriou, M. and M. Dasygenis, 2016. Teddy bear upgraded with an embedded system to react on feelings. Proceedings of the 5th International Conference on Modern Circuits and Systems Technologies, May 12-14, IEEE Xplore Press, Thessaloniki, Greece, pp: 1-4. DOI: 10.1109/MOCAST.2016.7495146

Jeong, H., D.P. Saakes and U. Lee, 2015. I-Eng: An interactive toy for second language learning. Proceedings of the 2015 ACM International Joint Conference on Pervasive and Ubiquitous Computing and International Symposium on Wearable Computers, Sept. 07-11, ACM, Osaka, Japan, pp: 305-308. DOI: 10.1145/2800835.2800857

Kara, N., C.C. Aydin and K. Cagiltay, 2014a. Design and development of a smart storytelling toy. Interactive Learn. Environ., 22: 288-297. DOI: $10.1080 / 10494820.2011 .649767$

Kara, N., C.C. Aydin and K. Cagiltay, 2014b. User study of a new smart toy for children's storytelling. Interactive Learn. Environ., 22: 551-563. DOI: $10.1080 / 10494820.2012 .682587$

Kickstarter. TROBO the Storytelling Robot, as Featured on TechCrunch. https://www.kickstarter.com/projects/trobo/trobo-thestorytelling-robot

Leal Penados, A., M. Gielen, P.J. Stappers and T. Jongert, 2010. Get up and move: An interactive cuddly toy that stimulates physical activity. Personal Ubiquitous Comput., 14: 397-406.

DOI: 10.1007/s00779-009-0270-3

Loujee.com. لوجي .. أول دمية عربية ذكية. http://loujee.com/

Low, S., Y. Sugiura, K. Fan and M. Inami, 2013. Cuddly: Enchant your soft objects with a mobile phone. Proceedings of the 10th International Conference on Advances in Computer Entertainment, Nov. 12-15, The Netherlands, pp: 138-151. DOI: 10.1007/978-3-319-03161-3 10

My Friend Cayla. This is Cayla. https://www.myfriendcayla.com/meet-cayla-c8hw 
Pham, D.M., T.N. Dam-Nguyen, P.T. Nguyen-Vo and M.T. Tran, 2013. Smart teddy bear a vision-based story Teller. Proceedings of the International Conference on Control, Automation and Information Sciences, Nov. 25-28, IEEE Xplore Press, Nha Trang, Vietnam, pp: 257-262.

DOI: 10.1109/ICCAIS.2013.6720564

Rafferty, L. and P.C.K. Hung, 2015. Introduction to Toy Computing. In: Mobile Services for Toy Computing, Hung, P.C.K. (Ed.), Springer, Cham, pp: 1-7.

Rafferty, L., B. Kroese and P.C.K. Hung, 2015. Toy Computing Background. In: Mobile Services for Toy Computing, Hung, P.C.K. (Ed.). Springer, Cham, pp: 9-38.

Smart Toy. Choose Your Own Adventure. https://www.smarttoy.com/cards
UC Davis Cancer Center and UC Davis Children's Hospital. Children learn through play. https://presidentscircle.childcare.utah.edu/_documen ts/children-learn-thru-play.pdf

Umaschi, M., 1997. Soft toys with computer hearts: Building personal storytelling environments. Proceedings of the Extended Abstracts on Human Factors in Computing Systems, Mar. 22-27, ACM, Atlanta, Georgia, pp: 20-21.

DOI: $10.1145 / 1120212.1120227$

Vaucelle, C. and T. Jehan, 2002. Dolltalk: A computational toy to enhance children's creativity. Proceedings of the Extended Abstracts on Human Factors in Computing Systems, Apr. 20-25, ACM, Minneapolis, Minnesota, USA, pp: 776-777. DOI: $10.1145 / 506443.506592$ 